\title{
Age-Related Hearing Loss in C57BL/6J Mice has both Frequency-Specific and Non-Frequency-Specific Components that Produce a Hyperacusis-Like Exaggeration of the Acoustic Startle Reflex
}

\author{
James R. Ison ${ }^{1,2}$, Paul D. Allen ${ }^{1}$, and William E. O’Neill ${ }^{1,2}$ \\ ${ }^{1}$ Department of Brain and Cognitive Sciences, University of Rochester, Meliora Hall, Rochester, NY 14627, USA \\ ${ }^{2}$ Department of Neurobiology and Anatomy, University of Rochester Medical Center, Rochester, NY 14642, USA
}

Received: 23 July 2007; Accepted: 27 August 2007; Online publication: 19 October 2007

\begin{abstract}
Auditory brainstem-evoked response (ABR) thresholds were obtained in a longitudinal study of C57BL/6J mice between 10 and 53 weeks old, with repeated testing every 2 weeks. On alternate weeks, acoustic startle reflex (ASR) amplitudes were measured, elicited by tone pips with stimulus frequencies of $3,6,12$, and $24 \mathrm{kHz}$, and intensities from subthreshold up to $110 \mathrm{~dB}$ sound pressure level. The increase in ABR thresholds for 3 and $6 \mathrm{kHz}$ test stimuli followed a linear time course with increasing age from 10 to 53 weeks, with a slope of about $0.7 \mathrm{~dB} /$ week, and for 48 $\mathrm{kHz}$ a second linear time course, but beginning at 10 weeks with a slope of about $2.3 \mathrm{~dB} /$ week. ABR thresholds for 12, 24, and $32 \mathrm{kHz}$ increased after one linear segment with a $0.7 \mathrm{~dB}$ slope, then after a variable delay related to the test frequency, shifted to a second segment having slopes of 3-5 $\mathrm{dB} /$ week. Hearing loss initially reduced the ASR for all eliciting stimuli, but at about 6 months of age, the response elicited by intense 3 and $6 \mathrm{kHz}$ stimuli began to increase to reach values about three times above normal, and previously subthreshold stimuli came to elicit vigorous responses seen at first only for the intense stimuli. This hyperacusis-like effect appeared in all mice but was especially pronounced in mice with more serious hearing loss. These ABR data, together with a review of histopathological data in the C57BL/ 6
\end{abstract}

Correspondence to: James R. Ison - Department of Brain and Cognitive Sciences - University of Rochester - Meliora Hall, Rochester, NY 14627, USA. Telephone: +1-585-2758461; fax: +1585-4429216; email: JIson@bcs.rochester.edu literature, suggest that the non-frequency-specific slow time course of hearing loss results from pathology in the lateral wall of the cochlea, whereas the stimulusspecific hearing loss with a rapid time course results from hair cell loss. Delayed exaggeration of the ASR with hearing loss reveals a deficit in centrifugal inhibitory control over the afferent reflex pathways after central neural reorganization, suggesting that this mouse may provide a useful model of age-related tinnitus and associated hyperacusis.

Keywords: aging, hearing loss, startle, plasticity, mixed strial/sensory presbycusis, tinnitus/hyperacusis

\section{INTRODUCTION}

This experiment in the C57BL/6J mouse provides a detailed analysis of the temporal course of age-related loss of hearing as captured by auditory brainstemevoked response (ABR) thresholds and its behavioral effects as observed in changes in the amplitude of the acoustic startle reflex (ASR). We used a longitudinal experimental design in which both the ABR and the ASR were tested every 2 weeks in a single group of mice between 2 months and 1 year of age. Mice of this inbred strain have been studied as an animal model for age-related progressive hearing loss resulting from peripheral sensorineural degeneration and, more recently, as a model for studying central neural reorganization and the behavioral consequences that follow the loss of afferent input from the auditory 
periphery. Their pattern of progressive hearing loss is generally reported as beginning at about 1 to 2 months with the highest spectral frequencies, which then increases in severity and spreads to lower frequencies with advancing age over the next 12 to 15 months. This outcome has been observed by many investigators using a variety of functional measures (Henry and Chole 1980; Shnerson and Pujol 1981; Willott 1986; Li and Borg 1991; White et al. 2000; Hequembourg and Liberman 2001; Ison and Allen 2003; and Mikaelian et al. 1974, in the related C57/ b16 strain of mice), but there is evidence in some reports of an additional earlier and relatively small non-frequency-specific or low-frequency hearing loss (Hequembourg and Liberman 2001; Ison and Allen 2003). The high-to-low frequency-specific pattern of hearing loss accords well with the progressive degeneration of hair cells along the basilar membrane as reported, for example, by Spongr et al. (1997), whereas the development of the early non-frequency-specific impairment may be attributed to the simultaneous advancing degeneration in the spiral ligament and stria vascularis observed by Hequembourg and Liberman (2001) before significant hair cell degeneration. There are other reports of age-related changes in stria vascularis, such as, for example, in the reduced density of its microvasculature and the loss of critical cell types, and in the changed expression of connexin 26 and of Na-K-ATPase in the lateral wall (Ichimiya et al. 2000; Di Girolamo et al. 2001; Ohlemiller et al. 2006), mostly at ages when they can be assumed to accompany sensorineural loss.

The central neural changes that accompany this advancing peripheral degeneration in the middleaged C57BL/ 6 mouse have been described by Willott (1996) as resulting in a widespread tonotopic reorganization that ultimately increases the low-frequency representation to invade much of the auditory cortex (Willott et al. 1993) and the inferior colliculus (Willott 1986) and appears also, but to a lesser extent, in the dorsal cochlear nucleus but not in the ventral cochlear nucleus (Willott et al. 1991). The behavioral effects that accompany this over-representation of low frequency stimuli include their providing stronger prepulse inhibition (PPI) of the ASR (Willott et al. 1994; Willott and Carlson, 1995; Carlson and Willott 1996) and also exaggerated ASR amplitudes (Ison and Allen 2003). Both sets of data are consistent with the position that high frequency hearing loss produces an increased salience of low frequency stimuli as a consequence of their increased tonotopic representation. Studying these neural and functional effects of the slow and cumulative progressive loss of peripheral afferent input with advancing age would appear to add a useful complement to the studies of the effects of the acute loss of hearing produced by the administra- tion of ototoxic drugs (Schwaber et al. 1993; Kakigi et al. 2000; Harrison 2001; Yang et al. 2007), mechanical damage (Robertson and Irvine 1989), or noise exposure (Salvi et al. 1996; Meleca et al. 1997): These studies similarly find a greater neural representation of stimulus frequencies that border the area of peripheral insult. Furthermore, an enhancement of late auditory-evoked potentials in the inferior colliculus and cortex for frequencies below the region of noise or salicylate-induced hearing loss has been reported (Salvi et al. 1996; Yang et al. 2007), which is thought to result from a change in the balance of central excitation and inhibition in local neural circuits bordering the region of hearing loss. The increase in the amplitude of auditory-evoked potentials observed in these experiments may be an electrophysiological counterpart of the exaggerated PPI and ASR found to accompany hearing loss in the C57BL/6J mouse.

Prior studies of changes in hearing sensitivity and associated changes in behavioral sensitivity in the aging C57BL/6 mouse have used cross-sectional studies that seem not well suited for describing the timing or generality of progressive degenerative effects that could be affected by the individual differences in hearing loss and neural reorganization that are known to exist even in inbred strains of mice (Willott et al. 1993). We believed that a longitudinal design with closely spaced repeated testing would better illustrate both the functional changes in hearing within and across frequencies with advancing age and the behavioral effects of hearing loss as seen in ASR amplitudes.

\section{MATERIALS AND METHODS}

\section{Subjects}

The group of subjects began with $16 \mathrm{C} 57 \mathrm{BL} / 6 \mathrm{~J}$ mice, 8 female and 8 male, bred in the University of Rochester Vivarium from parent stocks obtained from the Jackson Laboratories (Bar Harbor, ME). Four died over the course of the 10-month-long study, and the final group included eight females and four males. The mice were same-sex grouphoused in a constant climate and a 12:12 h normal light/dark cycle and tested in the daytime. Food and water were available ad libitum save during the test session. The ambient noise level in the colony room was $40 \mathrm{~dB}$ sound pressure level (SPL) at $2 \mathrm{kHz}$ and decreased linearly to $25 \mathrm{~dB}$ SPL at $24 \mathrm{kHz}$ on a logfrequency scale. All procedures were approved by the University of Rochester Committee on Animal Resources and were in accord with the regulations of the Public Health Service and the federal Animal Welfare Act. 


\section{Apparatus and procedures}

ABR recording. Mice were lightly anesthetized (2,2,2 tribromomethanol [Aldrich], at an intraperitoneal dose of $0.42 \mathrm{mg} / \mathrm{g}$, provided in $0.024 \mathrm{ml} / \mathrm{g}$ of a sterile saline solution) then placed on a 10-cm high platform inside a small $(57 \mathrm{~L} \times 41 \mathrm{~W} \times 36 \mathrm{H} \mathrm{cm})$, electrically shielded, soundproofed booth (IAC, Inc.) lined with 4.5-cm thick echo-attenuating acoustic foam (Sonex ${ }^{\circledR}$; Illbruck Acoustic, Minneapolis, MN). The mouse was arranged in a prone position facing a broadband electrostatic loudspeaker system (TDT ES1 with ED1 speaker driver) located $10 \mathrm{~cm}$ from the head and was kept warm during the procedure with a circulating-water heating pad. Miniature subdermal needle electrodes (Nicolet) were inserted at the vertex (reference), over the bulla (active), and just above the hind limb (ground). Calibrated tone bursts $(5 \mathrm{~ms}$ duration, $0.5 \mathrm{~ms}$ rise-fall time, phase alternating $90^{\circ}$ ) were synthesized with SigGenRP ${ }^{\odot}$ software on a TDT RP2.1 real-time processor and presented at a rate of $49 / \mathrm{s}$ at frequencies of 3,6 , 12, 24, 32, and $48 \mathrm{kHz}$. ABR waveforms were averaged in response to 300 tone bursts $(150 \times 2$ blocks $)$ at each tested frequency/amplitude combination. At each frequency, the amplitude of the signal was automatically attenuated (TDT PA5) in $5 \mathrm{~dB}$ steps between samples from $90 \mathrm{~dB}$ SPL (but $80 \mathrm{~dB}$ SPL maximum at $48 \mathrm{kHz}$ ) until the wave IV-V complex of the averaged ABR waveform was no longer distinguishable by eye from background in traces recorded with filter settings of 3 to $1,000 \mathrm{~Hz}$. By convention, threshold was established as the response at $5 \mathrm{~dB}$ above this final level. On rare occasions, thresholds could not be recorded for a particular frequency because the level of anesthesia was wearing off, and two test days were missed for one subgroup because of scheduling conflicts. For the day-to-day analyses of the overall data, the missing values that were specific to particular frequencies and specific mice were estimated at the mean of the two adjoining test days. Certain amplitude measures were later analyzed from these records after high pass filtering through a Butterworth zerophase digital filter with a $500 \mathrm{~Hz}$ cutoff frequency. Through the first 4 months of the experiment, ABR waveforms were obtained with a TDT System 2evoked potential workstation running BioSigRP ${ }^{\mathbb{C}}$ software (Tucker-Davis Technologies, Alachua, FL) on a Dell personal computer. The equipment was then upgraded to a System 3 workstation in which ABR waveforms were recorded (gain 5,020×) with a TDT RA4LI low-impedance digital headstage and RA4PA Medusa preamp controlled by a RA16 digital signal processor.

ASR measurements. Mice were tested in a wire cage, oval in shape and $5 \mathrm{~cm}$ wide, $7 \mathrm{~cm}$ long, and $4 \mathrm{~cm}$ high. The cage was mounted on a suspended acrylic platform to which an accelerometer was attached directly below the test cage. It was placed within a sound attenuating IAC room lined with sound-absorbing foam (inside dimensions approximately $2 \times 2 \times 2 \mathrm{~m}$ ). The force of the startle reflex was transduced by the accelerometer, its signal amplified and routed to an A/D converter (TDT RP2) then integrated using custom software over a $100 \mathrm{~ms}$ period beginning with stimulus onset. ASRs were elicited by tone pips ( $25 \mathrm{~ms}$ duration including $5 \mathrm{~ms}$ rise/fall times) varying in frequency $(3,6,12$, and $24 \mathrm{kHz})$ and in level (a $0 \mathrm{~dB}$ activity baseline trial, then six stimulus levels from 60 to $110 \mathrm{~dB}$ SPL in $10 \mathrm{~dB}$ steps). These stimuli were provided by a real-time digital signal processor (TDT RP2) attenuated by a programmable attenuator (TDT PA5), then amplified and delivered to a Yamaha Professional Series Compression tweeter. The ambient noise level in the chamber was $<25 \mathrm{~dB}$ SPL for all frequencies above $125 \mathrm{~Hz}$. Sound levels were measured with a $0.25^{\prime \prime}$ $(6.4 \mathrm{~mm}) \mathrm{B} \& \mathrm{~K}$ microphone (Model 4135) and sound level meter (Model 2203). The speaker was mounted directly over the center of the test cage at a distance of $15 \mathrm{~cm}$. The mouse was free to move within the cage, but was generally oriented along the major axis. During calibration, the microphone was placed at the position of the mouse ear for one orientation, the values being the same for one orientation or the other.

\section{Experimental design}

The mice entered the experiment after an initial ABR test at about 7 weeks of age (mean 49.2 days, $\mathrm{SD}=2.4$ ) administered as a screening examination to ensure that no mice began with poor hearing compared to colony records. Experimental testing began when the mice were 10 weeks old, and on this first test, the median change from the pretest was $0 \mathrm{~dB}$ at every frequency, and the most deviant mean change was $1.25 \mathrm{~dB}(\mathrm{SD}=3.77)$ for the $32 \mathrm{kHz}$ stimulus. The mice were then tested on a repeating fortnightly schedule from 10 to 53 weeks of age, save for weeks 18 and 22 when the ABR apparatus was unavailable because of the equipment upgrade and for maintenance. One half of the mice began the cycle with ABR testing, the others with ASR testing. The ABR test week also included a distortion product otoacoustic emission (DPOAE) test, the results of which will not be reported here. The ASR test was given every 2 weeks in a single session lasting about $75 \mathrm{~min}$. The session consisted of eight blocks of trials, with each block consisting of all 28 stimulus conditions presented in random order: four stimulus frequencies of $3,6,12$, and $24 \mathrm{kHz}$, each at seven levels $-0 \mathrm{~dB}$ (to provide a baseline activity score), and 60, 70, 80, 90, 100, and $110 \mathrm{~dB}$. Trials were separated on average by $20 \mathrm{~s}$, range 15 to $25 \mathrm{~s}$. 
The ABR thresholds and the ASR amplitudes of the two subgroups were aggregated in fortnightly bins, which for graphic and regression analyses, were nominally set at the mean age of the two subgroups (i.e., 10.5, 12.5 weeks, etc.). Age and stimulus frequency and stimulus level were subjected to inferential tests where appropriate, using analysis of variance (ANOVA; SPSS version 14) and the Huynh and Feldt (1976) correction for nonhomogeneity of inter-condition correlations in repeated-measures designs. Linear regression analyses were used to describe age-related changes in the ABR measures, and Pearson product-moment correlations were used to assess the strength of the association between individual differences in the ASR and ABR measures. These analyses and the graphic displays used Graphpad ${ }^{\circledR}$ software, version 4.0. The measures of effect size in terms of "proportion of variance accounted for" by any combination of independent variables were partial-etasquare $\left(\eta_{\mathrm{p}}^{2}\right)$ for the ANOVA from SPSS and $R^{2}$ for $t$ tests and correlation analyses.

\section{RESULTS}

\section{ABR thresholds}

The mean (SD) initial ABR thresholds (dB SPL) at 7 weeks of age were as follows: $3 \mathrm{kHz}, 50.8(5.6) ; 6 \mathrm{kHz}$, $25.4(5.8) ; 12 \mathrm{kHz}, 7.9$ (3.3); $24 \mathrm{kHz}, 17.5$ (7.2); $32 \mathrm{kHz}$, 22.7 (6.2); $48 \mathrm{kHz}, 25.4$ (4.5). Before this experiment, there had been $66 \mathrm{C} 57 \mathrm{BL} / 6$ mice tested between 6 and 8 weeks of age, with subgroups acquired from Harlan Sprague Dawley Inc. $(n=13)$ and from The Jackson Laboratory $(n=23)$, as well as those born in the vivarium $(n=30)$. Overall, the colony mean (SD) thresholds across frequencies were for $3 \mathrm{kHz}, 45.2 \mathrm{~dB}$ (9.2); $6 \mathrm{kHz}, 29.6 \mathrm{~dB}$ (8.5); $12 \mathrm{kHz}, 18.3 \mathrm{~dB}(7.2)$; $24 \mathrm{kHz}, 37.2 \mathrm{~dB}$ (20.5); $32 \mathrm{kHz}, 45.5 \mathrm{~dB}$ (23.0); and $48 \mathrm{kHz}, 51.3 \mathrm{~dB}$ (21.7). Spongr et al. (1997) saw small areas of hair cell degeneration at 4 weeks of age in C57BL/ 6 mice purchased from Charles River and Harlan Sprague Dawley Inc., their observation suggesting that hearing loss is already present in some C57BL/6J mice at a very early age: This seems consistent with the large variation found between mice at high test frequencies in our overall colony data and also with the similarly large standard deviations obtained in the colony of $\mathrm{Li}$ and Borg (1991) for 1-month-old mice. Some portion of this high variability in our data can be attributed to the diverse origin of the mice, as the ANOVA of the summed ABR thresholds showed that the subgroups were significantly different, $F(2 / 63)=38.56, p<0.001$, $R^{2}=0.58$. The colony-bred mice were not significantly different from the mice obtained from The Jackson Laboratory (Bonferroni multiple comparison test, $p>0.05)$, but both had lower thresholds than the mice obtained from Harlan Sprague Dawley, Inc. $(p<0.001)$. When the summed ABR values at 7 weeks of age for the present cohort were compared with the distribution of the Jackson and the Rochesterbred mice combined with their values in dB SPL ranked from low ("good hearing") to high ("poor hearing"), the cohort median was at the 83rd percentile, and even the mouse with the worst threshold values had better hearing than the colony median, falling at the 57 th percentile. Thus, we may conclude that the C57BL/6J mice we studied were relatively homogeneous in having excellent hearing, comparable to mice of about the same age tested by Hequembourg and Liberman (2001), but with better hearing than those tested by Li and Borg (1991), by White et al. (2000), and by Henry (2002).

Figure 1 shows the mean (SEM) loss of hearing sensitivity with increasing age across frequencies, quantified as the difference between each subject's ABR thresholds at each test age and their initial pretest value: This graph thus depicts a measure of hearing loss comparable to $\mathrm{dB}$ hearing level. The solid lines depict the results of linear regression analyses across different segments of these data. These analyses suggest that the group mean growth of age-related hearing loss is captured by single linear functions for 3,6 , and $48 \mathrm{kHz}$ and with the concatenation of two linear segments for the remaining frequencies. Hearing loss for $48 \mathrm{kHz}$ had a fast-rising slope (SE slope, $R^{2}$ ) of $2.26 \mathrm{~dB} /$ week $(0.06,0.99)$ beginning at 10 weeks of age, whereas hearing loss for 3 and $6 \mathrm{kHz}$ combined (their

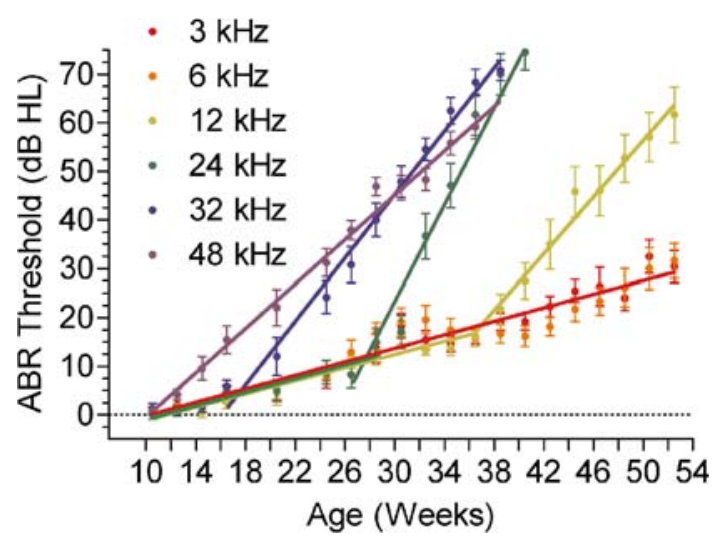

FIG. 1. The age-related development of hearing loss across tonal frequency for a group of $12 \mathrm{C} 57 \mathrm{BL} / 6 \mathrm{~J}$ mice tested every 2 weeks between the 10 and 53 weeks of age. Hearing loss was calculated as the difference ( $\mathrm{dB}$ SPL) in thresholds at any age and the thresholds obtained on a pretest at 7 weeks of age. The symbols provide the mean (SEM) at each age, and the regression lines provide the best estimate of the rate of hearing loss at different frequencies and ages. Depending on the test frequency, the rate of hearing loss with advancing age can be gradual or rapid or abruptly shift from slow to rapid. 
separate lines being close to identical) had a slowly rising slope of $0.69 \mathrm{~dB} /$ week $(0.04,0.95)$. Remarkably, the group mean hearing loss functions for the remaining frequencies of 12,24 , and $32 \mathrm{kHz}$ all began with their initial segments having slopes similar to those obtained for the 3 and $6 \mathrm{kHz}$ stimuli, which varied between 0.68 and $0.73 \mathrm{~dB} /$ week. All of the second segments had a rapidly rising slope like that of the $48 \mathrm{kHz}$ stimulus, but having significantly different values: for $12 \mathrm{kHz}, 2.91 \mathrm{~dB} /$ week $(0.16$, $0.98)$, for $24 \mathrm{kHz}, 5.10 \mathrm{~dB} /$ week $(0.40,0.96)$; and for $32 \mathrm{kHz}, 3.26 \mathrm{~dB} /$ week $(0.13,0.99)$. The calculated intersections of these two segments revealed that the second segment began at week 17 for the $32 \mathrm{kHz}$ stimulus, at week 28 for the $24 \mathrm{kHz}$ stimulus, and at week 37 for the $12 \mathrm{kHz}$ stimulus.

The slopes of the rapidly rising functions depicted in the group means of Figure 1 may result in part from the dispersion across subjects in the age at which the shift from the slow to the fast segment first appeared and in part from ensuing individual differences in the growth rate and severity of hearing loss. To examine these possibilities, we constructed Figure 2 to present the age-related change in ABR thresholds at $12 \mathrm{kHz}$ for each mouse. These individual functions are noisy, but the pattern is consistent with the group data in having a slowly increasing initial phase followed by a second phase in which hearing loss increased more rapidly. The estimated transition point from the first to the second process varied across mice by about 14

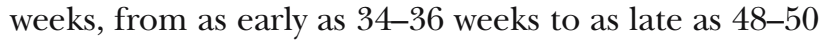
weeks of age.

It is of some interest that the two mice showing the most rapid loss of hearing in this figure were males, but the mouse with the most delayed loss of hearing was also a male. A test of the null hypothesis that hearing loss did not vary between males and females was accomplished by comparing their overall average

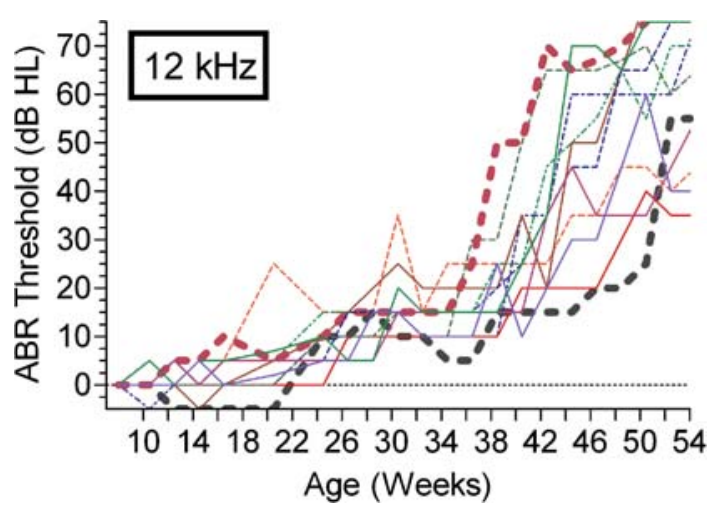

FIG. 2. The development of hearing loss with age in each mouse for the test stimulus of $12 \mathrm{kHz}$, showing inter-subject variability in the severity of hearing loss and in the age at which the slow development of hearing loss shifted to a rapid rate. hearing loss for each frequency. The ANOVA of these data provided a significant effect for frequency, $F(5 /$ $50)=112.02, p<0.001, \eta_{\mathrm{p}}^{2}=0.92$, but the interaction of sex and frequency was not significant, $F<1, \eta_{\mathrm{p}}^{2}=0.07$. The mean (SD) overall threshold was 33.6 (3.89) dB for the males and 30.2 (2.61) dB for the females, but the main effect of sex was not significant, $F(1 /$ $10)=3.41, p=0.10, \eta_{\mathrm{p}}^{2}=0.25$. However, because we lost subjects during the course of the experiment, the power to reject the null hypothesis for this comparison was only 0.39 . This plus the fact that the sex difference did account for $25 \%$ of the variance in these data suggests that we should acknowledge the presence of a small though not significant trend in these data showing that these female C57BL/6J mice may have a reduced rate of hearing loss compared to males. However, we note that Henry (2002) reported that the female C57BL/6J mice he tested, which were at least 200 days old, had significantly poorer high frequency thresholds than males of the same age.

To determine the degree of consistency of individual differences between the mice in their rate of high frequency hearing loss across test stimuli, an overall correlation was calculated for their hearing losses at each frequency on the test day at which the mean hearing loss for the group approximated $35 \mathrm{~dB}$ (for $48,32,24$, and $12 \mathrm{kHz}$ in order, weeks $24,26,32$, and 42). This correlation was positive but not significant: $R^{2}=0.20, p=0.16$. A comparable analysis of the low frequency data obtained when the mice were 1 year old found that individual differences in the ABR for 3 and $6 \mathrm{kHz}$ test stimuli were significantly correlated, $r=0.68, R^{2}=0.46, p=0.015$.

\section{ASR amplitudes}

The mean ASR amplitude for each subject for each nonzero stimulus condition on every test day was calculated as the difference between their mean recorded values for the various stimulus conditions and their mean baseline no-stimulus activity level on that test day. Figure 3 provides the mean (SEM) startle amplitudes at 2-week intervals across the entire experiment, for every frequency $(3,6,12$, and $24 \mathrm{kHz})$ and every level, from 60 to $110 \mathrm{~dB}$ SPL. The striking feature apparent in this family of ASR functions is the presence of strong interactions between the effects of the eliciting stimulus conditions and the age of the mouse and, overall, an apparent partition of the data into three more-or-less distinct processes. The first was a general decline in the ASR starting from their initial levels at 10-14 weeks of age when the $12 \mathrm{kHz}$ stimulus was the most effective, to a low point around 22 to 24 weeks of age when the differential effects of eliciting stimulus frequency and level were much reduced. In the second phase, lasting for about 14 to 16 weeks, the 

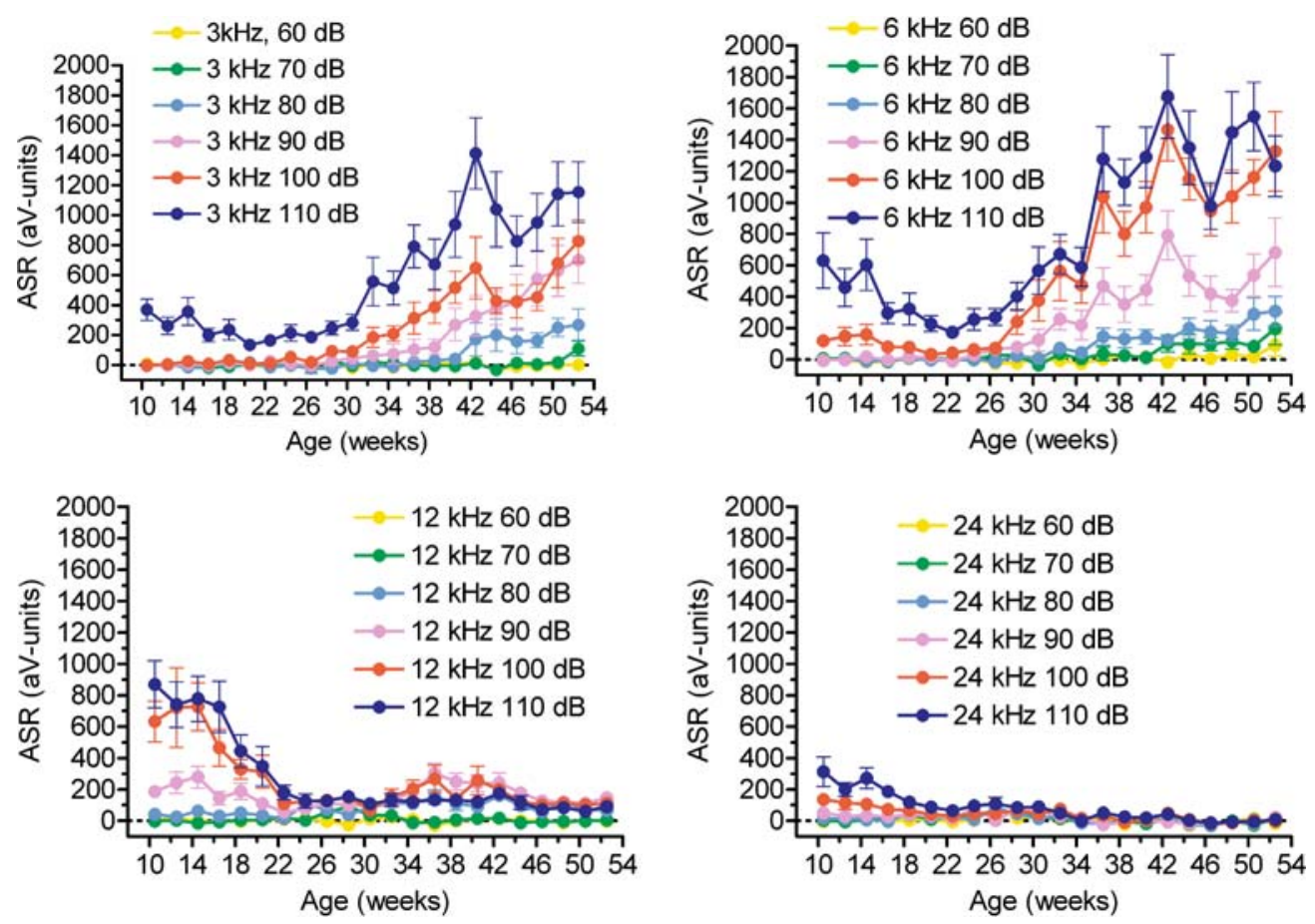

FIG. 3. Mean (SEM) ASR amplitudes across frequency, level, and age for the group of $12 \mathrm{C} 57 \mathrm{BL} / 6 \mathrm{~J}$ mice tested every 2 weeks between the 10 and 53 weeks of age, showing an early decrement in ASR amplitudes that affected all test frequencies with increased age and

ASR elicited by the 3 and $6 \mathrm{kHz}$ tone pips markedly increased and started to appear even at stimulus levels initially below the ASR threshold. In the last third of the experiment, after the age of about 40 weeks, the ASR amplitudes for the more intense levels of the 3 and $6 \mathrm{kHz}$ tone pips appeared to have stabilized, but with advancing age, the progression of startle responses elicited by less intense and previously subthreshold stimuli continued. During the middle phase and continuing through the end of the experiment, the ASR elicited by 12 and $24 \mathrm{kHz}$ tone pips stabilized at low but nonzero levels for the $12 \mathrm{kHz}$ tone pip and was not different from the background no-stimulus level for the $24 \mathrm{kHz}$ tone pip. However, there was a small temporary increase in the ASR elicited by the $12 \mathrm{kHz}$ stimulus when the mice were about 36 to 42 weeks of age.

These ASR data were subjected to an overall ANOVA in which, for simplicity, the age dimension was condensed to just three levels, early (average across the ages of 10 to 22 weeks), middle (24 to 38 weeks), and late ( 40 to 52 weeks). Consistent with the strength of the overall effects apparent in Figure 3 and described above, the ANOVA provided significant $F$ values $(p<0.001)$ for each of these main effects and for all of their interactions, including the three-way interaction of frequency, level, and age, $F(30 / 300)=27.89$, $p<0$. 001, $\eta_{\mathrm{p}}^{2}=0.74$. However, there was no significant main effect for sex, $F<1, \eta_{\mathrm{p}}^{2}=0.003$, and none of the interactions between sex, and age, frequency, or level

initial high frequency hearing loss, then a later progressive increase in the ASR confined to the lower tonal frequencies, but spreading from intense eliciting stimuli to formerly subthreshold stimuli as hearing loss became more severe and widespread.

approached significance (all $p>0.25$ ). Subsequent paired $t$-tests within and between these phases (reported here just at the $110 \mathrm{~dB}$ level) showed that the decrements in the ASR from weeks 10 to 22 were significant at each frequency: for $3,6,12$, and $24 \mathrm{kHz}$ in order, $p=0.007, R^{2}=0.50 ; p=0.02, R^{2}=0.41 ; p=0.0008$, $R^{2}=0.65$; and $p=0.02, R^{2}=0.41$. In sharp contrast, paired $t$-tests comparing the average ASR in the late phase of the experiment (ages from 40 to 52 weeks) with the initial ASR at 10 to 14 weeks of age showed significant increases for the 3 and $6 \mathrm{kHz}$ eliciting stimuli $\left(p=0.0004, R^{2}=0.69 ; 0.0003,0.71\right)$ but still significant decrements for the 12 and $24 \mathrm{kHz}$ stimuli $(p=0.004$, $\left.R^{2}=0.69 ; 0.007,0.50\right)$. The median proportionate ASR losses between the ages of 10 and 22 weeks for the 3,6 , 12 , and $24 \mathrm{kHz}$ stimuli in order were $0.58,0.63,0.81$, and 0.79 . The proportionate ASR increases for the 3 and $6 \mathrm{kHz}$ stimuli between 10 weeks of age and the terminal level of the ASR at 40 to 52 weeks of age were 3.08 and 2.67, whereas the proportionate ASR decrements for the 12 and $24 \mathrm{kHz}$ stimuli over this time were 0.89 and 0.98 .

Overall, these analyses of the entire group of mice indicate first that between 10 to 22 weeks of age, their ASR levels significantly declined in strength for every eliciting stimulus frequency; second that this low level of ASR expression persisted for the 12 and $24 \mathrm{kHz}$ stimuli through to the end of the experiment; and third, in contrast, that after 26 weeks of age, the ASR 
for the 3 and $6 \mathrm{kHz}$ stimuli increased to asymptotic levels two to three times more vigorous than those evident at the beginning of the experiment. Figure 4 provides scatter plots of ASR levels of individual mice at two ages. In the upper plot (Fig. 4a) the data were combined across all frequencies to show the general relationship between their early mean ASR at 10 to 14 weeks of age on the abscissa and their subsequent reduced mean ASR at 16 to 22 weeks on the ordinate. In the lower plot (Fig. 4b), the mean ASR amplitudes summed over 100 and $110 \mathrm{~dB}$ for the 3 and $6 \mathrm{kHz}$ frequencies are shown for their initial levels 10 to 14 weeks of age on the abscissa and their late response means over 24 to 52 weeks of age shown on the ordinate. The dotted lines provide the $Y=X$ function, whereas the solid line depicts the calculated regression line. It is noteworthy that, between the ages of 10-14
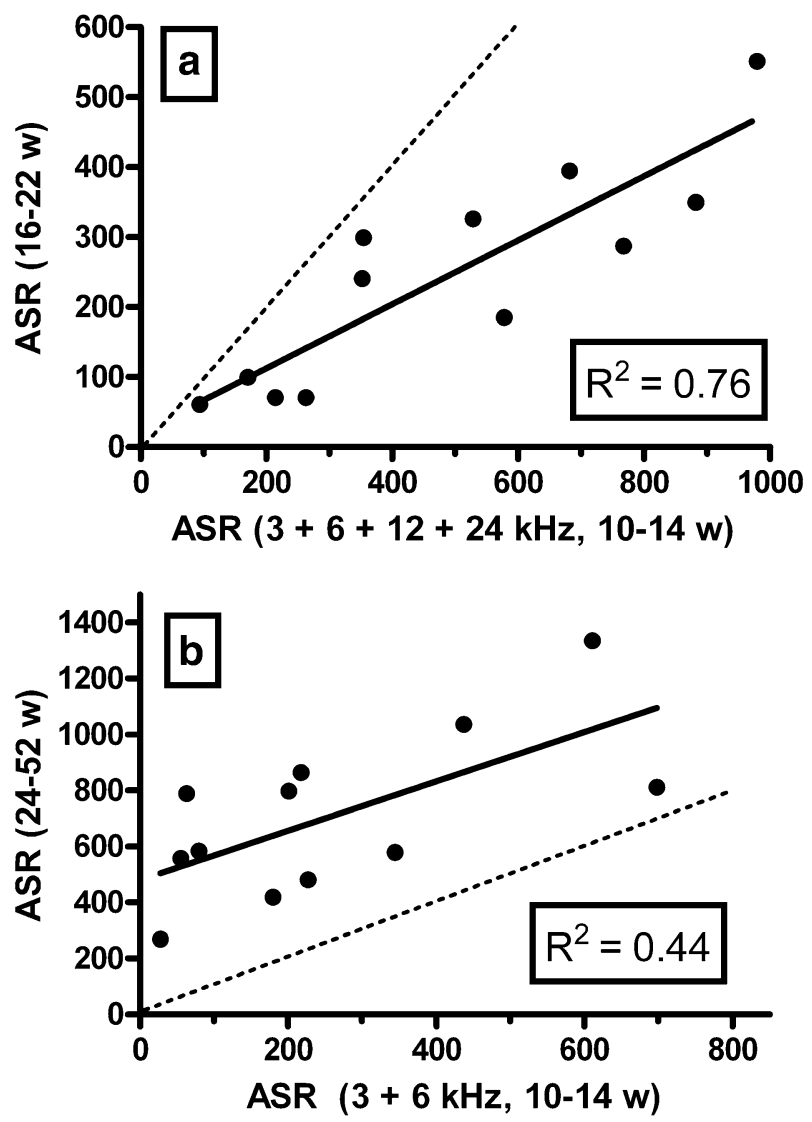

FIG. 4. a Scatter plots showing for each mouse the mean amplitude of the ASR combined over test frequencies of 3, 6, 12, and $24 \mathrm{kHz}$ at 100 and $110 \mathrm{~dB}$, on the $X$-axis for the first 3 weeks of the experiment and for the next 4 weeks on the $Y$-axis. b Scatter plots showing for each mouse the mean amplitude of the ASR combined over test frequencies of 3 and $6 \mathrm{kHz}$ at 100 and $110 \mathrm{~dB}$ for the first 3 weeks of the experiment on the $X$-axis and for the final 30 weeks of the experiment on the $Y$-axis. These data show that the early cross-frequency ASR decrements and the later low frequencyspecific increments in the ASR were present in all mice, but the individual differences in the ASR between mice at these different ages were highly correlated. and 16-22 weeks, the ASR decreased in every mouse and that, between the ages of 10-14 and 24-52 weeks, the ASR for 3 and $6 \mathrm{kHz}$ combined increased in every mouse. The regression line $Y=0.46 X+21$ accounted for $76 \%$ of the variance in the early decrement of the ASR $(p=0.0002)$, whereas the regression line $Y=0.88 X+480$ accounted for $44 \%$ of the variance in the late increment in the ASR $(p<0.02)$.

Individual differences in the onset or severity of hearing loss and the ASR

The ASR data for the individual mice shown in Figure 4 indicate that individual differences in the strength of the ASR amplitudes were moderately stable across the course of this experiment, as their responses when they were 16 to 22 weeks and also 24 to 52 weeks of age were both significantly correlated with their initial response levels when they were 10 to 14 weeks old. The stability of these individual differences in ASR across this substantial age difference suggests that ABR expression as an individual trait must be relatively uniform with increasing age, even at an age of 1 year compared to 2 months. We note that the remaining "unaccounted-for" variance in these data could be the result of random variation and measurement error, or possibly, of individual differences in hearing loss and/ or its more central effects. To examine the relationship between individual differences in hearing loss and individual ASR levels, the residual values from the regression analyses of the ASR data obtained in the first period of hearing loss (weeks 16-22) and in the subsequent periods of the development and maintenance of exaggerated responding (weeks 24 52) that could not be accounted for by the initial response differences were further analyzed to see if they were related in some degree to individual differences in hearing loss. The primary results of these analyses are shown in the scatter plots of Figure 5, with the levels of the ASR after the early initial ASR decrement in the upper plot (Fig. 5a) and the later increase in low frequency ASR levels shown in the lower plot (Fig. 5b). The individual differences in the ASR residuals obtained when the mice were 16 to 22 weeks old had near zero correlations with individual differences in hearing loss for either 48 or $32 \mathrm{kHz}$ stimuli between 10 and 22 weeks of age, but there was a marginally significant positive correlation $(r=+0.56$, $R^{2}=0.32, p=0.056$ ) between these residuals and the mean hearing loss at $3,6,12$, and $24 \mathrm{kHz}$ over the same ages. The individual differences in the late ASR levels at 3 and $6 \mathrm{kHz}$ that were not accounted for by the initial differences in the ASR were found to be positively correlated with the combined hearing loss for 12 and $24 \mathrm{kHz}$ between the ages of 10 and 52 weeks of age $\left(r=+0.59, R^{2}=0.35, p=0.043\right)$. Both of the 

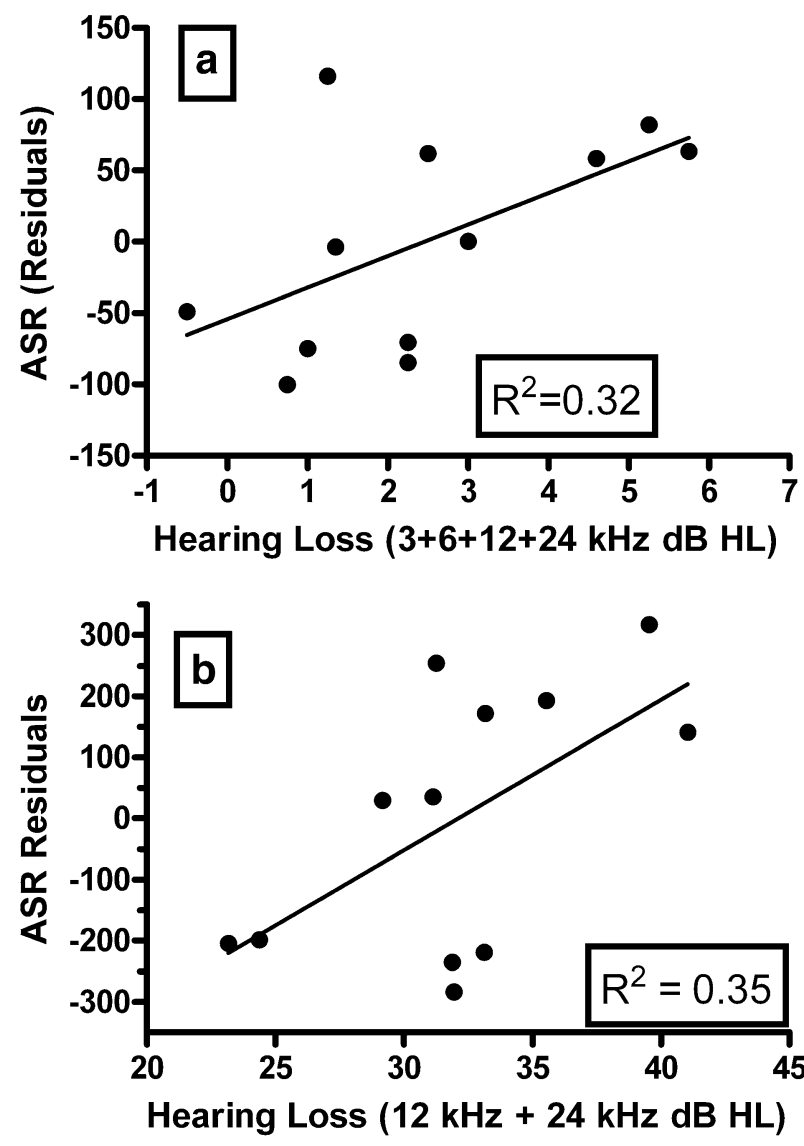

FIG. 5. a Scatter plots showing for each mouse on the $X$-axis, the mean amplitude of the combined hearing loss over test frequencies of 3,6, 12, and $24 \mathrm{kHz}$ during the first 7 weeks of the experiment and on the $Y$-axis, the residual deviations from the regression line shown in Figure 4a. b Scatter plots showing for each mouse on the $X$-axis, the mean amplitude of the combined hearing loss over test frequencies of 12 and $24 \mathrm{kHz}$ over the entire duration of the experiment and on the $\mathrm{Y}$-axis, the residual deviations from the regression line shown in Figure $4 \mathrm{~b}$. These calculations suggest that $\mathrm{a}$ more severe hearing loss was associated with a greater ASR than predicted from the initial ASR level, given the regression lines shown in Figure 4.

regression lines shown in Figure 5 indicate that the mice with greater threshold hearing loss responded more vigorously than was predicted given their initial ASR level. It is interesting too that the temporary increase in the $12 \mathrm{kHz}$ ASR at 36 to 42 weeks of age coincided with the transition to the more rapid rate of hearing loss for $12 \mathrm{kHz}$ and followed near complete hearing loss at the higher tonal frequencies, but we did not find significant correlations in individual differences in the ASR and the ABR measures.

In addition to the threshold hearing loss shown in Figure 1, ABR amplitudes (in microvolts) were tabulated from the peak of $\mathrm{P} 1$ to the trough between $\mathrm{P} 1$ and $\mathrm{P} 2$ for the $90 \mathrm{~dB}, 6 \mathrm{kHz}$ test stimulus on weeks 10 , 26, and 52. The resulting mean (SD) values descended in order as $3.05(1.30), 2.87(0.80)$, and 1.76 (0.82): The ANOVA of these data provided a significant age effect, $F(2 / 22)=5.04, \quad p=0.017$, $\eta_{\mathrm{p}}^{2}=0.31$. These amplitude measures were not significantly correlated with the standard ABR thresholds measured in dB SPL: for 10, 26, and 52 weeks in order, $R^{2}=0.05,0.07,0.00$. However, at 10 weeks of age, the $6 \mathrm{kHz}$ ABR amplitude was significantly correlated with the amplitude of the ASR for the $110 \mathrm{~dB} 6 \mathrm{kHz}$ eliciting stimulus $\left(r=+0.64, R^{2}=0.40\right.$, $p=0.026$ ), but at 26 weeks of age, the correlation was near zero $(r=+0.02)$, whereas at 52 weeks of age, the correlation, while not significant, was negative $\left(r=-0.32, R^{2}=0.10, p=0.30\right)$.

\section{DISCUSSION}

Two processes with different time constants describe $\mathrm{C} 57 \mathrm{BL} / 6$ J hearing loss

Variation in the rate of hearing loss across age and test frequency shown in the ABR thresholds of Figure 1 is similar to the findings reported by others in crosssectional studies (particularly, e.g., Hequembourg and Liberman 2001), but the precision afforded in this repeated measurement design provides a strong confirmation of these earlier findings and certainly greater temporal detail. These data suggest that hearing loss in the C57BL/6J mouse is produced by two types of degeneration that vary in their rate of development and in their cochlea targets. One type produces an apparent uniform decrement across all test frequencies that gradually increases in severity over the first year of life; the second yields a superimposed more rapid increase in severity that appears at earlier ages for higher frequency stimuli. A similar two-segment effect with increasing age is apparent in a cross-sectional comparison presented by Hequembourg and Liberman (2001). Their data show that, across the ages of 1.5, 3 , and 7 months of age, there was a linear increase in ABR thresholds for both 7 and $22.6 \mathrm{kHz}$ test stimuli of about $0.6 \mathrm{~dB}$ per week (these values estimated from their Fig. 10, page 128). This slow progression was maintained for the $7 \mathrm{kHz}$ test stimulus, but for the $22.6 \mathrm{kHz}$ stimulus, it was replaced by a more rapid process that resulted in a severe hearing loss of about $50 \mathrm{~dB}$ that was incurred sometime before the final test at 15 months of age. The related histopathological analyses depict a widespread and progressive loss of type IV fibrocytes of the spiral ligament and a steadily decreasing width of the stria vascularis apparent as early as 3 months of age. In sharp contrast, there was little change in spiral ganglion cells in the 6-week to 15-month comparison and very little outer hair cell loss evident in the cytocochleogram until the final test age and then only in the $22 \mathrm{kHz}$ region of the basilar membrane. We should note, however, that more subtle structural changes, for example, in the normal 
conformation of the stereocilia, would be invisible to this analysis, but, at least, we can anticipate that these effects would be also frequency specific.

These observations support the conjecture that, in this mouse, the cochlear pathology responsible for the initial slowly increasing hearing loss that appears to be uniform across frequencies, at least from 3 to $32 \mathrm{kHz}$, is in the spiral ligament and the stria vascularis, whereas the subsequent rapid loss of hearing for specific frequencies results from the loss of functioning hair cells along the basilar membrane. Both of these pathologies are found in postmortem analysis of the ears of presbycusic human listeners (Nelson and Hinojosa 2006) just as Mikaelian et al. (1974) had observed them both to be present in the old C57/b16 mouse, but an unanswered question has been the relative ages at which these two pathologies and their dissimilar functional consequences first appear. The complex two-stage pattern of age-related progressive hearing loss evident in the ABR data presented here, allied with the similar pattern and the histopathological findings of Hequembourg and Liberman (2001), provides a plausible answer to this question, at least for the C57BL/6J mouse. However, the unsolved challenge to this plausible conjecture is to identify the physiological mechanism by which this known strial histopathology adversely affects hearing, given the two recent reports that the endocochlear potential (EP) is maintained throughout the life span of the C57BL/6J mouse (Lang et al. 2002; Ohlemiller et al. 2006), even in the presence of massive strial atrophy. These two reports appear to provide strong evidence against the hypothesis that "metabolic presbycusis" is in part responsible for early-onset age-related hearing loss in our mice. One implication of this discrepancy between these evidently normal EP measures and both the compelling histopathological findings and the associated pattern of hearing loss is that the pathways intervening between strial pathology and non-frequency-specific age-related hearing loss may not yet be fully understood. For an example of the apparent complexity of these pathways, there are the provocative data presented by Spiess et al. (2002) of the effects on cochlea function of uncoupling gap junctions by proadifen. In one gerbil, they reported that the acute administration of a low dose of this compound to the round window substantially reduced the amplitude of compound action potentials evoked by high tonal frequency stimuli but had no effect on DPOAE amplitudes: This suggestion of a direct effect on inner hair cells was accompanied by only a small decrement in the EP. We recall that Ichimiya et al. (2000) had observed a reduced density of connexin 26 staining in the spiral ligament in $\mathrm{C} 57 \mathrm{BL} / 6$ mice between 6 months and 1 year of age: These two reports suggest the value of further study of these pathways in the aging mouse.

\section{ASR amplitudes can be both reduced and exaggerated by hearing loss}

The initial behavioral consequence of the highfrequency hearing loss at $48 \mathrm{kHz}$ and the beginning of hearing loss at $32 \mathrm{kHz}$ was a loss of ASR amplitudes for all eliciting stimuli, although the loss was somewhat greater for the 12 and $24 \mathrm{kHz}$ startle-eliciting stimuli compared to 3 and $6 \mathrm{kHz}$. Parham and Willott (1988) had similarly observed that, in C57BL/6J mice, ASR thresholds to mid-frequency-eliciting stimuli were raised more than could be expected on the basis of deficits in their frequency-specific hearing thresholds. They forwarded the reasonable hypothesis that the ASR/ABR threshold disparity may have resulted at least in part at a peripheral level, because in the young mouse, the ASR to a nominally "low tonal frequency" startle-eliciting stimulus would be increased by the spread of excitation along the basilar membrane to the more sensitive higher frequency regions of the basilar membrane: This spread of excitation necessarily would have less behavioral impact after high frequency hearing loss. Their hypothesis is similarly congruent with our observation that a startle-eliciting stimulus of $6 \mathrm{kHz}$ is more effective than is $24 \mathrm{kHz}$ but has a higher ABR threshold. It agrees also with our finding in the young mouse a positive correlation between the amplitudes of the $6 \mathrm{kHz}$ ASR and the suprathreshold $\mathrm{P} 1-\mathrm{N} 1$ amplitudes of the ABR to a $6 \mathrm{kHz}$ test stimulus, whereas neither of these two response measures correlate with the ABR thresholds at $6 \mathrm{kHz}$. These data suggest that, for relatively intense low tonal frequencies, both ASR expression and ABR amplitudes are enhanced by the apical spread of excitation before but not after high frequency hearing loss.

Liberman and Dodds (1984) have shown that the low-frequency tails of tuning curves of high tonal frequency inner haircells become supersensitive after outer hair cell loss. That the amplitude of the ABR for suprathreshold low tonal frequencies declines with advancing age and hearing loss in the mouse suggests that this peripheral type of supersensitivity is not a significant factor in determining the suprathreshold ABR amplitude. In addition, finding that the initial positive correlation between the ASR and suprathreshold ABR amplitudes for the $6 \mathrm{kHz}$ test stimuli disappears and may be even negative in the 1year-old mouse further indicates that ASR enhancement is not driven by an increased signal from the auditory nerve, but from the delayed secondary consequences of its having provided a diminished signal after hearing loss. 
The importance of high frequency hearing loss for an early age-related ASR decrement in the C57BL/6J mouse is further revealed in contrasts with the CBA mouse, with no hearing loss and no early ASR decrement (Parham and Willott 1988; Ison and Allen 2003), and with the DBA/2 mouse, with its more serious early hearing impairment and an accelerated ASR decrement (Willott et al. 1984). It thus may have been expected a priori that individual differences in the early ASR decrement would be correlated with individual differences in hearing loss at 48 or $32 \mathrm{kHz}$, but the obtained correlations were near zero. However, one limiting factor in our using regression analysis to identify possible "causative" variables for these behavioral phenomena is that it relies on the presence of individual differences in behavior within the group that may then be found to be correlated with individual differences in hearing loss. In the present data sets, the mice were relatively homogeneous, the initial ASR decrement and the subsequent ASR increments were present in every mouse, and individual differences in the later ASR values were largely accounted for by their differing initial values at the beginning of the experiment: Thus, in comparison with the less homogeneous groups of mice tested by Ison and Allen (2003), here we had relatively little additional "unexplained" variance that could be correlated with differences in hearing loss. The failure to find any effect of variation in the extent of high frequency hearing losses and the ASR at 16 to 22 weeks of age may be best interpreted as indicating that, by this age, every mouse had incurred a significant high frequency hearing loss. However, one suggestive effect of hearing loss on the magnitude of the ASR at this early age appeared in a post hoc and only marginally significant correlation between the ASR residuals and the magnitudes of the small across-frequency hearing loss common to 3,6 , 12 , and $24 \mathrm{kHz}$ ABR test frequencies combined. The results of this analysis suggested that the greater this common hearing loss, then the more vigorous was the obtained ASR compared to the predicted level given the regression equation and the initial response amplitude.

Additional regression analyses were performed to determine if individual differences in the development or in the terminal levels of the exaggerated ASR obtained at 3 and $6 \mathrm{kHz}$ could be accounted for by differences in hearing loss. Like the outcome for the initial decrement in the ASR, the late ASR for all mice was exaggerated compared to the initial level, but the initial level differences between mice accounted for much of the variance in the late levels. The analyses of the "non-explained" differences in the degree of ASR exaggeration between mice found that the overall hearing losses at 32 and $48 \mathrm{kHz}$ correlated with behavior at near zero levels, but the ASR residuals were significantly correlated with individual differences between mice in their hearing loss at 12 and $24 \mathrm{kHz}$ combined. This analysis also showed that mice with greater hearing loss responded more vigorously than would be predicted by the regression equation and the initial ASR data.

\section{SUMMARY AND CONCLUSIONS}

Evidence for a persistent form of non-frequency-specific hearing loss

Beginning with the seminal research of Mikaelian et al. (1974) and confirmed by many subsequent experiments, the most striking feature of the phenomenology of age-related hearing loss in the C57BL/6J model has been its progressive high to low frequency pattern. Some irregular exceptions to this basic pattern have been noted (this perhaps not being surprising, given the usual small sample size in these experiments and the actual degree of variability typically obtained within an inbred strain), but these have been most noticeable at early ages. The results obtained in the present longitudinal study with its frequent and regular testing schedule confirm the presence of this initial non-frequency-specific form of hearing loss and extend the prior observations in showing that it is not restricted to just low tonal frequencies or just young ages. In these data, we found the slowly developing form of hearing loss evident at some ages across all frequencies between at least 3 and $32 \mathrm{kHz}$, and it continued for low frequency test stimuli until the mice were 12 months of age. It was only the age of the mouse at the time of the transition from the slowly developing to the rapidly developing process that followed the familiar path of high to low frequency hearing loss. This two-stage progression of age-related hearing loss is at least qualitatively similar to the two-stage progression from non-regional-specific degeneration in the lateral wall and stria vascularis to the later regional and frequency-specific degeneration of the outer hair cells reported by Hequembourg and Liberman (2001). These data suggest the C57BL/6J mouse as a potentially useful animal model for studying the longitudinal progression of mixed sensory/strial presbycusis.

\section{Evidence for changes in the balance of neural excitability with hearing loss}

The early behavioral consequence of high-frequency hearing loss is a near uniform ASR decrement for all eliciting stimuli, a common finding that suggests that these relatively intense stimuli (at least, relative to 
absolute thresholds) normally provide an apical spread of excitation along the basilar membrane that provides significant excitatory drive for the startle reflex pathways, at least until the onset of high frequency hearing loss. The delayed ASR increase for low frequencyeliciting stimuli seen here and in the earlier only partly longitudinal experiment (Ison and Allen 2003) is consistent with the behavioral and physiological observations of Willott and his colleagues (reviewed in Willott 1996), that low-frequency tone pips provide more PPI of the ASR, and that they have also a greater neural representation in the dorsal cochlear nucleus, in the inferior colliculus, and in auditory cortex. However, although these structures include the afferent pathways for PPI, the sensory and motor pathways for the primary ASR are in the cochlear root and the ventral cochlear nucleus, that are the apparent origin of the direct axonal connections to the pontine motor nuclei (Koch 1999). These more caudal structures are unlikely to have a greater representation of low frequency stimuli after high frequency hearing loss (Willott et al. 1991). We thus suggest that the greater sensitivity to low frequency startle-eliciting stimuli in the primary reflex pathways must result as a secondary consequence of the loss of normal downstream centrifugal control from the more rostral centers in which reorganization after hearing loss is a prominent feature. We hypothesize that this phenomenon of ASR exaggeration is conceptually similar to the enhanced auditory-evoked potentials appearing very soon after noise exposure or salicylate administration in the inferior colliculus and the auditory cortex (Salvi et al. 1996; Yang et al. 2007), save that it appears only after a substantial delay that may be necessary for the neural reorganization of the more rostral sites that normally modulate startle expression.

Discussions of the possible implications for human auditory experience of these demonstrations of neuroplasticity after hearing loss in the C57BL/6J mouse (for example, Willott 1996) suggest the possibility that tonotopic reorganization may provide a compensatory benefit, as shown in human listeners by, for example, Thai-Van et al. (2003), but also that it may produce perceptual distortion or tinnitus. These sensory penalties of reorganization might occur, for example, if perceived pitch remained attached to particular high frequency central locations that, after reorganization, were broadly and erroneously excited by lower frequency stimuli or, if the loss of central inhibition increased spontaneous activity in still functional areas that had lost their normal patterns of innervation. Kaas (1996) has pointed to the inappropriate localization of tactile stimuli and the illusory experience of persistent and distressing "phantom" stimulation resulting from limb amputation as untoward effects of somatosensory neuroplasticity that provide dire predictions as to the potential outcomes of neuroplasticity after hearing loss. The exaggerated startle reactions to low frequency stimuli in the $\mathrm{C} 57 \mathrm{BL} / 6 \mathrm{~J}$ mouse may be analogous to hyperacusis, the increased aversion to normally insignificant sounds that often accompanies tinnitus in human listeners (Nelson and Chen 2004). Noise exposure and ototoxic drugs are two common antecedents of tinnitus in humans, and so, it is not surprising that these are also the two manipulations most often used to induce hearing loss and then tinnitus in laboratory animals. But although there is a striking increase in the prevalence of tinnitus with advancing age (Henry et al. 2005), we are not aware of any research in animals that has established a link between age-related hearing loss and tinnitus. Given recent advances in the development of screening tests for tinnitus in rodents using a simple gap detection paradigm, both in an operant conditioning procedure (Prosen and May 2005) and with startle reflex inhibition audiometry (Turner et al. 2006; Yang et al. 2007), it is possible that further research with the C57BL/6J mouse will establish its value for the study of hyperacusis and tinnitus as well as agerelated hearing loss and neuroplasticity.

\section{ACKNOWLEDGEMENTS}

The work reported here was supported by US Public Health Service NIH Grants, AG095247 and DC05409. We are grateful to John Housel for collecting the ABR measures.

\section{REFERENCES}

Carlson S, Willott JF. The behavioral salience of tones as indicated by prepulse inhibition of the startle response: relationship to hearing loss and central neural plasticity in C57BL/6J mice. Hear. Res. 99:168-175, 1996.

Di Girolamo S, Quaranta N, Picciotti P, Torsello, A, Wolf F. Agerelated histopathological changes of the stria vascularis: an experimental model. Audiol. 40:322-326, 2001.

HARRISON RV. Age-related tonotopic map plasticity in the central auditory pathways. Scan. Audiol. 30:8-14, 2001.

Henry KR. Sex- and age-related elevation of cochlear nerve envelope response (CNER) and auditory brainstem response (ABR) thresholds in C57BL/6 mice. Hear. Res. 170:107-115, 2002.

Henry KR, Chole RA. Genotypic differences in behavioral, physiological and anatomical expressions of age-related hearing loss in the laboratory mouse. Audiol. 5:369-383, 1980.

Henry JA, Dennis KC, Schechter MA. General review of tinnitus: prevalence, mechanisms, effects, and management. J. Speech Lang. Hear. Res. 48:1204-1235, 2005. 
Hequembourg S, Liberman MC. Spiral ligament pathology: a major aspect of age-related cochlear degeneration in C57BL/6 mice. JARO 2:118-129, 2001.

Huynh H, Feldt LS. Estimation of the Box correction for degrees of freedom from sample data in randomized block and split-plot designs. J. Educ. Stat. 1:69-82, 1976.

Iсніміуa I, Suzuki M, Mogi G. Age-related changes in the murine cochlear lateral wall. Hear. Res. 139:116-122, 2000.

Ison JR, Allen PD. Low-frequency tone pips elicit exaggerated startle reflexes in C57BL/6J mice with hearing loss. JARO 4:495-504, 2003.

KAAS JH. Plasticity of sensory representations in the auditory and other systems of adult animals. In: Salvi RJ, Henderson D, Fiorino F, Colletti V (eds) Auditory System Plasticity and Regeneration. NY Thieme Medical Publishers, Inc. pp. 213-223, 1996.

Kakigi A, Hirakawa H, Harel N, Mount RJ, Harrison RV. Tonotopic mapping in auditory cortex of the adult chinchilla with amikacin-induced cochlear lesions. Audiol. 39:153-160, 2000.

Косн M. The neurobiology of startle. Progr. Neurobiol. 59:107-128, 1999.

Lang H, Schulte BA, Schmiedt RA. Endocochlear potentials and compound action potential recovery: functions in the C57BL/ 6J mouse. Hear. Res. 172:118-126, 2002.

Li HS, Borg E. Age-related loss of auditory sensitivity in two mouse genotypes. Acta Oto-Laryngol. 111:827-834, 1991.

Liberman MC, Dodds LW. Single-neuron labeling and chronic cochlear pathology. III. Stereocilia damage and alterations of threshold tuning curves. Hear. Res. 16:55-74, 1984.

Meleca RJ, Kaltenbach Ja, Falzarano PR. Changes in the tonotopic map of the dorsal cochlear nucleus in hamsters with hair cell loss and radial nerve bundle degeneration. Brain Res. 750:201213, 1997.

Mikaelian DO, Warfield D, Norris BA. Genetic progressive hearing loss in the $\mathrm{C} 57 / \mathrm{b} 16$ mouse: Relation of behavioral responses to cochlear pathology. Acta Otolaryngol. 77:327-333, 1974.

Nelson JJ, Chen K. The relationship of tinnitus, hyperacusis, and hearing loss. Ear Nose Throat J. 83:472-476, 2004.

Nelson EG, Hinojosa R. Presbycusis: a human temporal bone study of individuals with downward sloping audiometric patterns of hearing loss and review of the literature. Laryngosc. 116:1-12, 2006.

Ohlemiller KK, Lett JM, Gagnon PM. Cellular correlates of agerelated endocochlear potential reduction in a mouse model. Hear. Res. 220:10-26, 2006.

Parham K, Willott JF. Acoustic startle response in young and aging C57BL/6J and CBA/J mice. Beh. Neurosci. 102:881-886, 1988.

Prosen C, May BJ. Behavioral and electrophysiological assessment of tinnitus in a mouse model. Ass. Res. Otolaryngol. Abstr. 28:\#410, 2005.

Robertson D, IRvine DRF. Plasticity of frequency organization in auditory cortex of guinea pigs with partial unilateral deafness. J. Comp. Neurol. 282:456-471, 1989.

Salvi RJ, Wang J, Powers N. Rapid functional reorganization in the inferior colliculus and cochlear nucleus after acute damage. In: Salvi RJ, Henderson D, Fiorino F, Colletti V (eds) Auditory
System Plasticity and Regeneration. NY, Thieme Medical Publishers, Inc. pp. 297-316, 1996.

SCHWABER MK, GarRaghty PE, KAAS JH. Neuroplasticity of the adult primate auditory cortex following cochlear hearing loss. Am. J. Otol. 14:252-258, 1993.

Shnerson A, Pujol R. Age-related changes in the C57BL/6J mouse cochlea. I. Physiological findings. Brain Res. 254:65-75, 1981.

Spiess AC, Lang H, Schulte BA, Spicer SS, Schmiedt RA. Effects of gap junction uncoupling in the gerbil cochlea. Laryngosc. 112:1635-1641, 2002.

Spongr VP, Flood DG, Frisina RD, Salvi RJ. Quantitative measures of hair cell loss in CBA and C57BL/6 mice throughout their life spans. J. Acoust. Soc. Am. 101:3546-3553, 1997.

Thai-Van H, Micheyl C, Moore BCJ, Collet L. Enhanced frequency discrimination near the hearing loss cut-off: a consequence of central auditory plasticity induced by cochlear damage? Brain 126:2235-2245, 2003.

Turner JG, Brozoski TJ, Bauer CA, Parrish JL, Myers K, Hughes LF, CASPARY DM. Gap detection deficits in rats with tinnitus: a potential novel screening tool. Beh. Neurosci. 120:188-195, 2006.

White JA, Burgess BJ, Hall RD, Nadol JB. Pattern of degeneration of the spiral ganglion cell and its processes in the C57BL/6J mouse. Hear. Res. 141:12-18, 2000.

WiLLOTT JF. Effects of aging, hearing loss, and anatomical location on thresholds of inferior colliculus neurons in C57BL/6 and CBA mice. J. Neurophys. 56:391-408, 1986.

WiLlott JF. Auditory system plasticity in the adult C57BL/6J mouse. In: Salvi RJ, Henderson D, Fiorino F, Colletti V (eds) Auditory System Plasticity and Regeneration. NY, Thieme Medical Publishers, Inc. pp. 297-316, 1996.

WiLlott JF, Carlson S. Modification of the acoustic startle response in hearing-impaired C57BL/6J mice: prepulse augmentation and prolongation of prepulse inhibition. Beh. Neurosci. 109:396-403, 1995.

Willott JF, Kulig J, SATterfield T. The acoustic startle response in DBA/2 and C57BL/6 mice: relationship to auditory neuronal response properties and hearing impairment. Hear. Res. 16:161-167, 1984.

Willott JF, Parham K, Hunter KP. Comparison of the auditory sensitivity of neurons in the cochlear nucleus and inferior colliculus of young and aging $\mathrm{C} 57 \mathrm{BL} / 6 \mathrm{~J}$ and $\mathrm{CBA} / \mathrm{J}$ mice. Hear. Res. 53:78-94, 1991.

Willott JF, Aitkin LM, McFadden SL. Plasticity of auditory cortex associated with sensorineural hearing loss in adult C57BL/6J mice. J. Comp. Neurol. 329:402-411, 1993.

Willott JF, Carlson S, Chen H. Prepulse inhibition of the startle response in mice: relationship to hearing loss and auditory system plasticity. Beh. Neurosci. 108:703-713, 1994.

Yang G, Lobarinas E, Zhang L, Turner J, Stolzberg D, Salvi R, Sun W. Salicylate induced tinnitus: Behavioral measures and neural activity in auditory cortex of awake rats. Hear. Res. 226:244-253, 2007. 